\title{
A Frequency control method for wind power system based on general energy storage model and particle swarm optimization
}

\author{
Qian Cheng',a, Qian Hai 2,b, \\ ${ }^{1}$ Guangzhou No.2 Middle School, Guangzhou 510000, Guangdong Province, China \\ ${ }^{2}$ CSG EHV Power Transmission Company, Guangzhou 510663, Guangdong Province, China \\ aquianhai@ehv.csg.cn, bqianhaigy@126.com \\ ${ }^{*}$ Corresponding author
}

\begin{abstract}
Keywords: Frequency control; Particle swarm optimization; Energy storage model; Wind farm. Abstract. Based on the frequency control of power system with wind turbine, this paper proposed a general model for energy storage system. On this basis, a method of power network frequency control based on particle swarm optimization (PSO) is proposed. By finding the optimal location of the particles, the optimal control parameters for the current grid are obtained. The performance of the proposed approach is tested on the IEEE-39 bus system. Experimental results indicate that the PSO algorithm can provide the optimal PI controller with proportional and integral parameters. The frequency fluctuation which is caused by wind farm can be obviously reduced. And the traditional problem of controller parameters misalignment can be solved.
\end{abstract}

\section{Introduction}

With the gradual depletion of fossil fuels, the sustainable energy capacity has been increased gradually. As a representative of sustainable energy, wind energy itself is random and intermittent[1]-[5]. This will impact the local power grid, so that the power quality of the power grid. Recent studies have shown that the combination of wind power and energy storage systems can effectively improve system transient stability. Energy storage system as a measure to suppress the system power fluctuations has become an important technical method to enhance the system and to absorb new energy capacity.

Literature [6] studied the principle of smooth control of wind energy storage system. By controlling the energy storage system smoothly, the high frequency fluctuation in the total output power of the scenery is suppressed. In literature [7], the damped torque analysis (DTA) method has been applied to complex multi machine systems including energy storage elements. The feasibility of the energy storage system tuning method is verified. In literature [8], a fuzzy control strategy of energy storage based on load level and wind speed is proposed when the transmission capacity of the line is limited. literature [9] proposes a series parallel parallel ultracapacitor system based on a unified control strategy to improve the stability of grid connected wind farm output. The energy storage system can smooth the power output of the wind farm under the strong wind speed disturbance, and reduce the impact of the wind farm on the grid. In literature [10], an autoregressive moving average wind speed forecasting model is established. Combined with the application of energy storage devices in power system, sequential Monte Carlo simulation method is used to evaluate the influence of the reserve capacity of wind farm, energy storage device and gas turbine on the reliability of generation and transmission system. These papers have studied the effectiveness of the energy storage system to suppress the power fluctuation of wind power grid through different strategies. But most of the above literatures are based on specific energy storage systems, and have strong pertinence.

In order to study the influence of different energy storage systems on electromagnetic transients, a general energy storage model based on data stream for electromechanical transient calculation of systems is proposed in this paper. In order to optimize the effect of the PI controller in the energy 
storage model, the particle swarm algorithm is used to adjust the proportion and integral parameters of the PI controller. The energy storage model is combined with the wind farm model[11]-[13].

By using the energy storage system to absorb and release energy in time, the fluctuation of wind farm output power is reduced, and the frequency stability of wind power grid is improved remarkably.

\section{Energy storage system model}

\subsection{Mathematical model of energy storage system}

As an important method to stabilize the new energy power fluctuation, the energy storage system is widely concerned and used. At present, energy storage technology can be divided into the following 3 categories according to the different forms of energy conversion. Mechanical energy storage is the first category which is represented by pumping energy storage, flywheel energy storage and compressed air energy storage. Electromagnetic energy storage is the second class which is represented by superconducting magnetic energy storage and ultracapacitor energy storage. Chemical energy storage is the third category which is represented by lead acid batteries, all vanadium redox flow batteries and sodium sulfur batteries. The differences between these specific energy storage systems are mainly embodied in the aspects of energy storage, response time delay, charge and discharge rate limits and energy storage capacity limitations. These characteristic parameters usually exist in the model in terms of constraints, and their mathematical models can be unified[14].

The structure of the common energy storage system can be divided into 3 parts: the energy storage component, the converter and the control system. The port characteristic of the grid connected to the energy storage system is closely related to the control strategy of the converter. The control strategy of converter is divided into two levels: outer loop control and inner loop control. External loop control is active power control. In the power system, the fluctuation of system frequency is mainly affected by the active power balance. The method of frequency control is used for the active power output of the energy storage system. The frequency deviation signal is used as the input signal. Through the control of the PI controller, a given value of power is generated Pset, which is shown as figure 1.

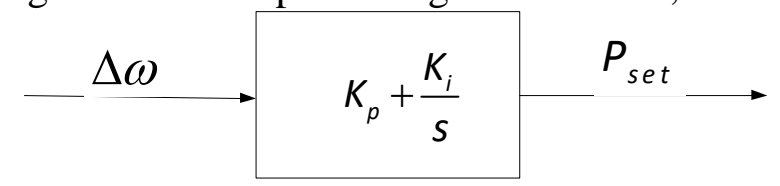

Fig. 1. block diagram of outer control

The active decoupling control is used in the inner loop control. Literature [11] adopts a feed-forward decoupling strategy. The current control equation after coupling is obtained. Then the transfer function of the current control equation is achieved. By means of the principle of mutual cancellation, the transfer function of the active power $\mathrm{P}$ of the energy storage device injection system is obtained. Its approximation is equivalent to first order inertial links, which is shown as follows:

$$
\varphi(s)=\frac{K}{1+T s}
$$

Where, $\mathrm{T}$ is the time constant, and $\mathrm{K}$ is the gain. By formula (1), the characteristic function of the power set point $P_{\text {set }}$ generated by the AC side of the current converter and the active power $\mathrm{P}$ of the energy storage device injected into the system can be obtained:

$$
P=-P / T+P_{\text {set }} / T
$$

In the real-time power regulation process of the energy storage system, the inner loop power control process can be simplified as a first order dynamic model, as shown in figure 2 .

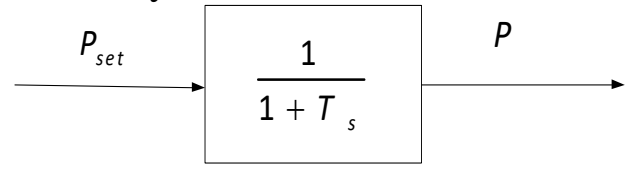

Fig.2 block diagram of inner control loop 
In practice, the energy storage system is expensive, so the capacity of the energy storage system must be limited[15]. For the energy storage system, the energy storage capacity cannot be released until the next time limit, and energy cannot be absorbed in the first time limit, which is shown as equation (3).

$$
S_{L}<\sum \Delta P \Delta t<S_{H}
$$

Where $S_{\mathrm{L}}$ is the lower limit of energy storage capacity. $\mathrm{S}_{\mathrm{H}}$ is the upper limit. $\Delta t$ is the power point sampling interval. In addition, the charge and discharge power of different energy storage systems is different. The charging and discharging power of the storage energy reflects the ability of the energy storage system to stabilize the power fluctuation of the wind power. Therefore, in the system simulation, different charging and discharging power values are set up to measure the effect of different energy storage systems.

\subsection{Simulink model of energy storage system}

Matlab / Simulink is a scientific computing software for matrix calculation, and has strong data processing ability. The mathematical model of the energy storage system in Simulink can be used to study the power fluctuation characteristics of the wind farm[16]. The mathematical model of the energy storage system built in Simulink is divided into 2 modules, which are active control output module and energy limiting link. The active control output module is shown in figure 3.

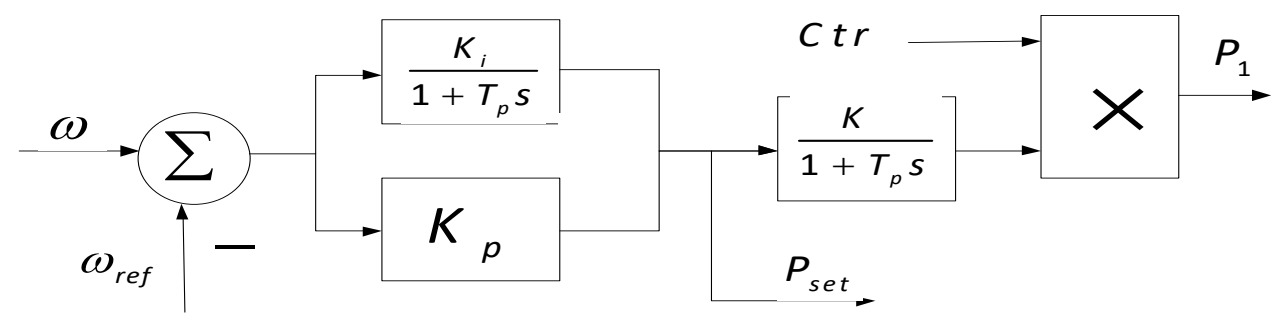

Fig.3 Active power output module

As shown in Figure 3, $\omega$ is Actual angular frequency of a fan, $\omega_{r e f}$ is fan reference angle frequency. $P_{1}$ is actual output power of fan. $P_{\text {set }}$ is given value of the active power of the fan. $C_{t r}$ is the control signal of the energy. VS modules are used to compare size relationships between input quantities.

\section{Particle swarm optimization (PSO) control strategy}

PSO algorithm has strong optimization ability for complex nonlinear problems. The bridge between the particle swarm algorithm and the Simulink model is the adaptive value of the particle and the particle (that is the performance index of the control system). The specific optimization process is as follows: PSO algorithm generates particle swarm, and the particles in the particle swarm are assigned to the parameters of PI controller $\mathrm{Kp}$ and $\mathrm{Ki}$. Then the Simulink model of the control system is employed and The performance metrics of this corresponding parameters is obtained. This performance index is passed into the PSO as an adaptive value of the particle. Update particle velocity and position using the following method:

$$
\begin{gathered}
x_{t+1}=\omega x_{t}+C_{t r 1}\left(P_{t}-x_{t}\right)+C_{t r 2}\left(G_{t}-x_{t}\right) \\
x_{t+1}=x_{t}+v_{t+1}
\end{gathered}
$$

Where, $x$ is the position of the particle, $v$ is the velocity of the particle; $w$ is the inertial factor; $C_{t r l}$ and $C_{t r 2}$ are the acceleration constants, $P_{t}$ is the best location that particles have searched, $G_{t}$ is the optimal location searched for the whole particle swarm so far.

Implementation steps for particle swarm algorithm:

1) Initializing step. The position and velocity of all particles are randomly generated. Then the parameters of $P_{t}$ and $G_{t}$ are obtained. 
2) For each particle, its fitness values are compared with the fitness of the optimum position $P_{t}$ experienced by the particle. If it better than current value, the $P_{t}$ is replaced by the new value.

3) For each particle, its fitness values are compared with the fitness is compared with the fitness of the optimal location $G_{t}$ experienced by the whole particle swarm,. If it better than current value, the $G_{t}$ is replaced by the new value.

4) Update the velocity and position of particles.

5) If the termination condition is not satisfied, return step 2. If the set error range or the maximum number of iterations is reached, the exit algorithm is used

\section{Case study}

The control effect on frequency and power of the wind farm has been verified in an energy storage system. In this paper, IEEE-39 bus system power system has been tested by the proposed method. Taking wind disturbance as an example, the operation characteristics of wind farm, the phase angle of generator bus voltage and the frequency fluctuation of generator nodes are simulated and analyzed. In this paper, the direct drive permanent magnet synchronous generators with wind power equivalent are added to the 30 bus in the IEEE-39 bus system. In simulation, the actual output power of fan is $1.5 \mathrm{MW}$. And the rated capacity of the whole wind farm is $200 \mathrm{MW}$. The internal resistance of equivalent synchronous wind generator is $0.8 \mathrm{pu}$. its mean to Ignore the influence of the internal resistance of the generator. The power factor of the equivalent direct drive synchronous generator is set as 0.95. The main parameters of the wind turbine are shown in Table [19].

Table 1. Wind Turbine Parameters

\begin{tabular}{cc}
\hline Wind turbine parameters & Figure \\
\hline Radius of wind wheel $/ \mathrm{m}$ & 37.5 \\
\hline Air density $/(\mathrm{kg} / \mathrm{m} 3)$ & 1.225 \\
\hline Rated wind speed $/(\mathrm{m} / \mathrm{s})$ & 13.1 \\
\hline Rated speed of wind turbine/(rad/s) & 1.7829 \\
\hline Optimum tip velocity ratio & 7.8 \\
\hline Maximum utilization factor of wind energy & 0.5 \\
\hline
\end{tabular}

In this case, a detailed model is adopted for the aerodynamic model. The wind speed change curve is from a real wind farm. At $\mathrm{t}=0$, the base wind with a wind speed of $9.8 \mathrm{~m} / \mathrm{s}$ is added throughout the simulation. Then the wind speed is changed dramatically. The maximum speed is $14.5 \mathrm{~m} / \mathrm{s}$. At the end of simulation time, the wind speed is dropped to normal value. The curve of wind speed is shown in figure 4.

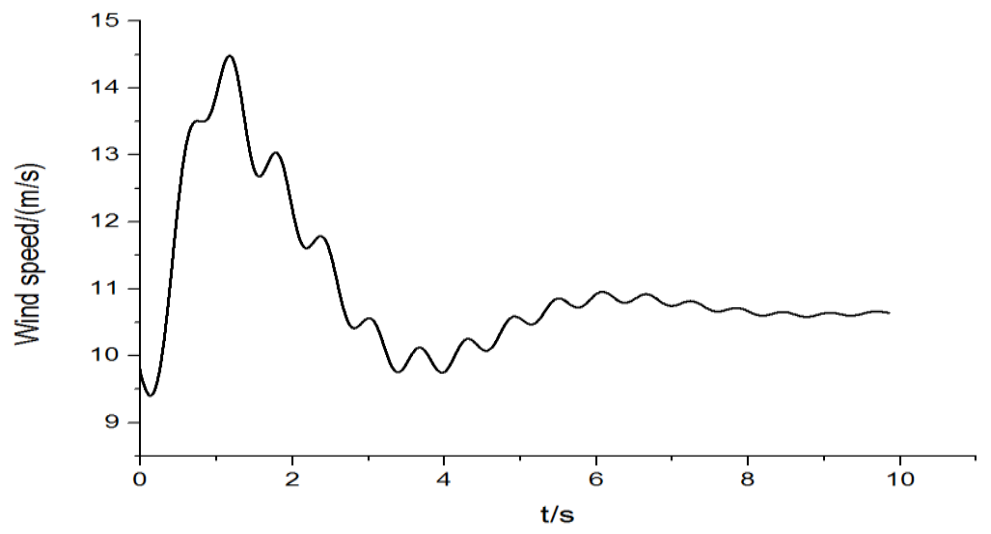

Fig.4 Curve of wind speed

When the wind speed changes from the rated wind speed to the gust state, the input power fluctuates accordingly. As can be seen from Figures 5 and 6, the system frequency recovers slowly. When the energy storage module is added, the system frequency oscillation is decreased, and the frequency fluctuation effect is suppressed. 
In this case, the energy storage system is added. When the energy storage systems are added, the frequency response and the phase difference increment of the grid are compared to verify the validity of the model. Since there are too many nodes, 12, 30 and 33 buses are used to represent non generator bus, fans bus and ordinary generator nodes. When the wind turbine is subjected to the disturbance mentioned above, the wind turbine system is tested. In this case, the discharging capacity and other characteristic parameters are set as the same. Two groups are employed to verify the effectiveness of proposed method. In group one, particle swarm optimization (PSO) is employed to control system power frequency. In group two, PSO is not used in the system. The phase angel increment based on the three kinds of bus type is shown in Figure $7-9$. As can be seen from figures $7-9$, when the proposed method is added, the output power fluctuation of the wind farm can be obviously improved.

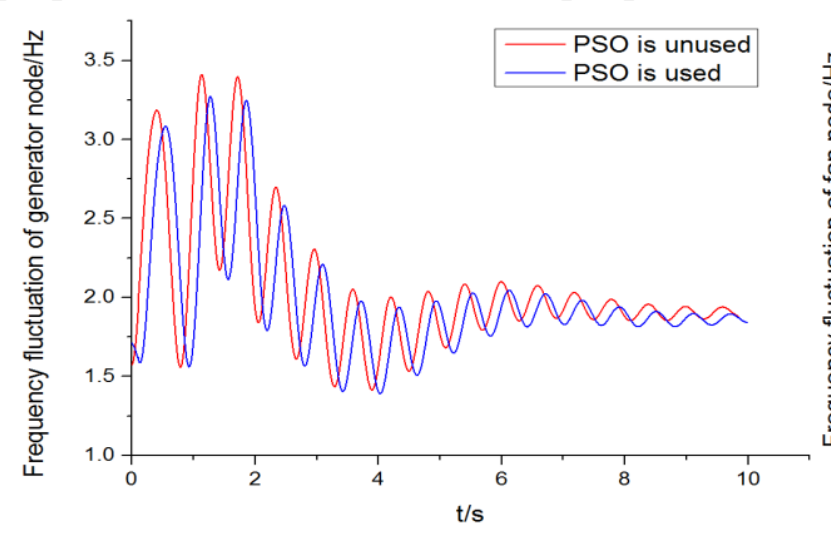

Fig. 5 Frequency fluctuations of generator

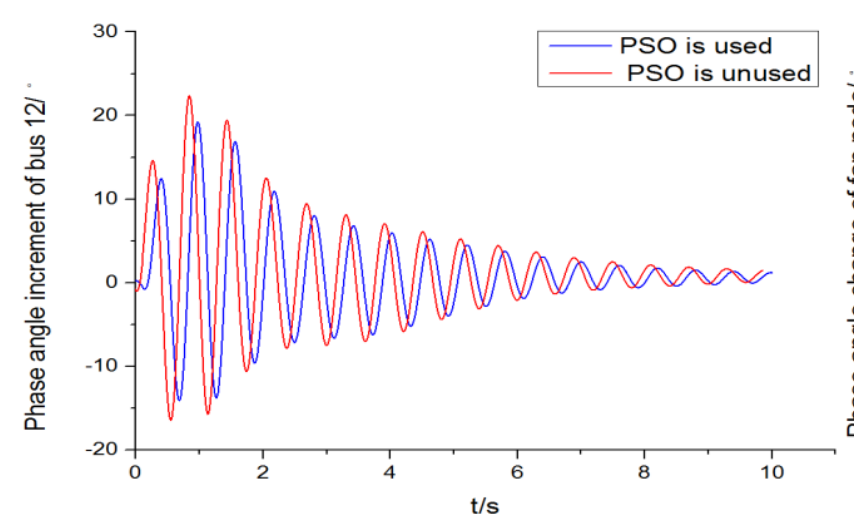

Figure 7 phase angel increment of bus 12

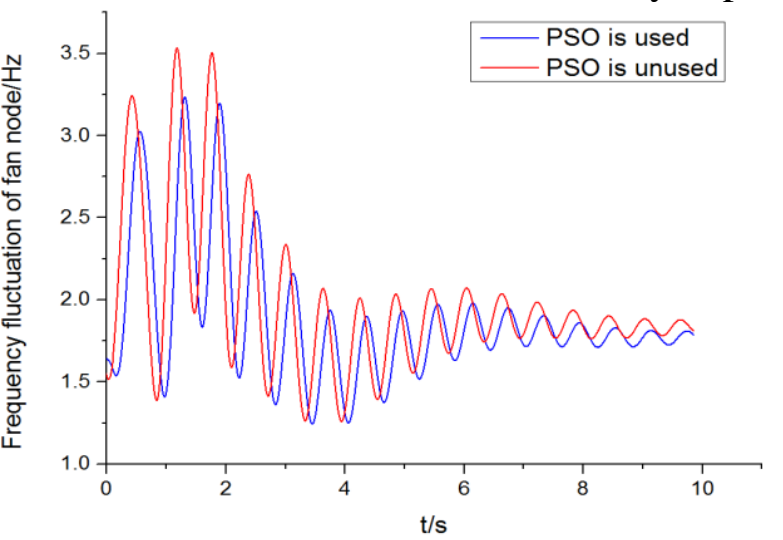

Fig. 6 Frequency fluctuations of fan

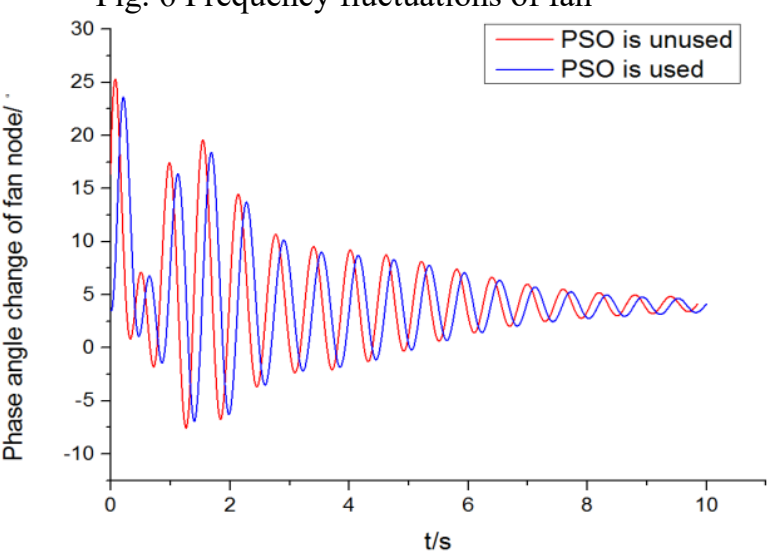

Figure 8 phase angel increment of fan

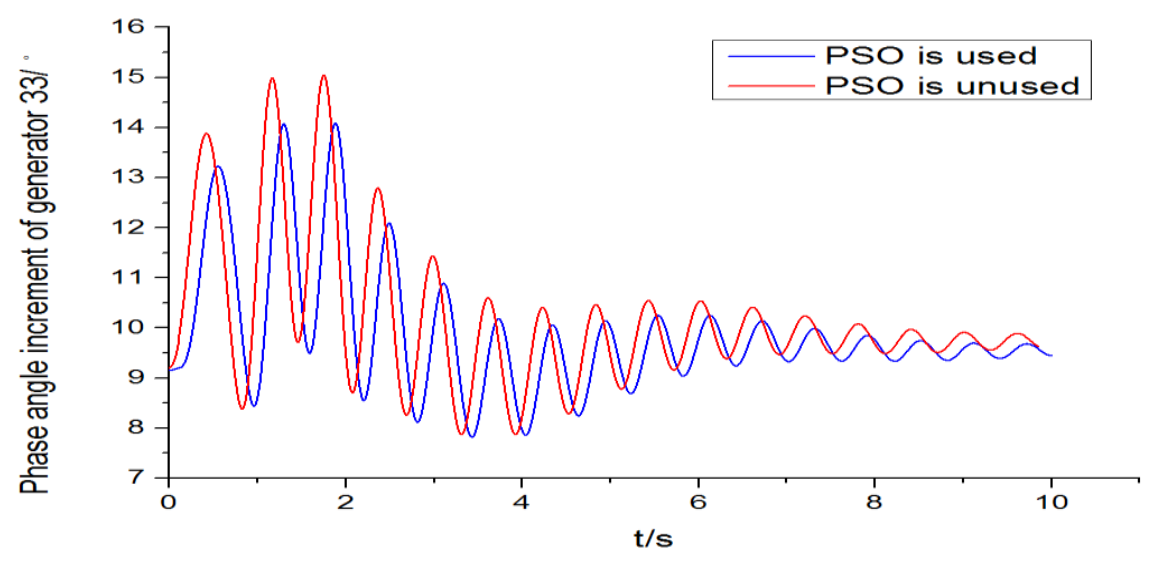

Figure 9 phase angel increment of generator

$$
c^{2}=a^{2}+b^{2} \text {. }
$$




\section{Conclusion Literature references}

In this paper, a mathematical model of general energy storage system is put forward and combined with wind farm. In the case of adding energy storage devices, the output power and frequency of the wind farm are analyzed by Simulink simulation. The result is as follows:

(1) PSO intelligent algorithm is used to optimize the energy storage system, The system can provide better dynamic stability control. The system can suppress the fluctuation of wind farm output power very well. Therefore, the system effectively solves the problem of randomness and volatility of wind power generation.

(2) PSO intelligent algorithm has been added to the energy storage device, which can be extended to photovoltaic power generation. In a specific working environment, PSO algorithm can provide the optimal PI controller with proportional and integral parameters. Therefore, the traditional problem of controller parameters misalignment can be solved.

\section{References}

[1] J. Tan and Y. Zhang, "Coordinated Control Strategy of a Battery Energy Storage System to Support a Wind Power Plant Providing Multi-Timescale Frequency Ancillary Services," in IEEE Transactions on Sustainable Energy, vol. 8, no. 3, pp. 1140-1153, July 2017.

[2] J. Hu, L. Sun, X. Yuan, S. Wang and Y. Chi, "Modeling of Type 3 Wind Turbines With df/dt Inertia Control for System Frequency Response Study," in IEEE Transactions on Power Systems, vol. 32, no. 4, pp. 2799-2809, July 2017.

[3] P. Tielens and D. Van Hertem, "Receding Horizon Control of Wind Power to Provide Frequency Regulation," in IEEE Transactions on Power Systems, vol. 32, no. 4, pp. 2663-2672, July 2017.

[4] W. Guo, F. Liu, J. Si, D. He, R. Harley and S. Mei, "Online Supplementary ADP Learning Controller Design and Application to Power System Frequency Control With Large-Scale Wind Energy Integration," in IEEE Transactions on Neural Networks and Learning Systems, vol. 27, no. 8, pp. 1748-1761, Aug. 2016.

[5] X. Liu, Y. Zhang and K. Y. Lee, "Coordinated Distributed MPC for Load Frequency Control of Power System With Wind Farms," in IEEE Transactions on Industrial Electronics, vol. 64, no. 6, pp. 5140-5150, June 2017.

[6] A. Ashouri-Zadeh, M. Toulabi and A. M. Ranjbar, "Coordinated design of fuzzy-based speed controller and auxiliary controllers in a variable speed wind turbine to enhance frequency control," in IET Renewable Power Generation, vol. 10, no. 9, pp. 1298-1308, 102016.

[7] Y. Liu, L. Jiang, Q. H. Wu and X. Zhou, "Frequency Control of DFIG-Based Wind Power Penetrated Power Systems Using Switching Angle Controller and AGC," in IEEE Transactions on Power Systems, vol. 32, no. 2, pp. 1553-1567, March 2017.

[8] D. Ochoa and S. Martinez, "A Simplified Electro-Mechanical Model of a DFIG-based Wind Turbine for Primary Frequency Control Studies," in IEEE Latin America Transactions, vol. 14, no. 8, pp. 3614-3620, Aug. 2016.

[9] W. Ren and E. Larsen, "A Refined Frequency Scan Approach to Sub-Synchronous Control Interaction (SSCI) Study of Wind Farms," in IEEE Transactions on Power Systems, vol. 31, no. 5, pp. 3904-3912, Sept. 2016. 